\title{
Abciximab-Associated Thrombocytopenia After Previous Tirofiban-Related Thrombocytopenia
}

\author{
Michael P. Dorsch, Pharm.D., Debbie Montague, M.S., Jo E. Rodgers, Pharm.D., and \\ Cam Patterson, M.D.
}

A 62-year-old man with a history of coronary artery disease and coronary artery bypass graft, chronic heart failure, and peripheral vascular disease required percutaneous coronary intervention (PCI) after progression of shortness of breath and fatigue over 2 years. Four hours after the procedure, the patient developed hematemesis and was found to be thrombocytopenic. The thrombocytopenia was presumed to be due to the abciximab infusion the patient received during and shortly after the PCI. Further review of the patient's medical history revealed that a similar episode had occurred 11 years earlier. At that time, he was enrolled in a clinical trial comparing tirofiban and heparin in patients with unstable angina; he developed profound thrombocytopenia within 24 hours of randomization. After the study unblinding, investigators discovered that the patient received tirofiban, which was thought to be the cause of his thrombocytopenia. Both abciximab and tirofiban are glycoprotein IIb-IIIa inhibitors, and thrombocytopenia induced by this class of drugs is a serious and potentially life-threatening adverse reaction. The mechanism is not well understood but has been described as immune mediated with both ligand-mimetic agents (tirofiban and eptifibatide) and abciximab. Our patient's situation was unusual in that he developed thrombocytopenia from a ligand-mimetic agent and subsequently had a similar reaction to abciximab. To our knowledge, this case report is the first documentation of thrombocytopenia associated with both tirofiban and abciximab in a single patient, and suggests that care should be given in administering glycoprotein IIb-IIIa inhibitors of either type to patients with a history of thrombocytopenia due to one of these agents.

Key Words: thrombocytopenia, glycoprotein IIb-IIIa inhibitor, GPIIb-IIIa inhibitor, tirofiban, abciximab, drug-induced thrombocytopenia.

(Pharmacotherapy 2006;26(3):423-427)

The glycoprotein IIb-IIIa inhibitors are a class of antithrombotic agents that act by inhibiting the platelet fibrinogen binding receptor, which in

From the Department of Pharmacy Services, University of Michigan Hospitals and Health Clinics, Ann Arbor, Michigan (Dr. Dorsch); and the Schools of Pharmacy (Ms. Montague and Dr. Rodgers) and Medicine (Dr. Patterson), University of North Carolina, Chapel Hill, North Carolina.

Address reprint requests to Michael P. Dorsch, Pharm.D., BCPS, Department of Pharmacy Services, University of Michigan Hospitals and Health Clinics, UH B2D 301/0008, 1500 East Medical Center Drive, Ann Arbor, MI 481090008; e-mail: mdorsch@med.umich.edu. turn inhibits platelet aggregation. Three agents are marketed in the United States: abciximab, eptifibatide, and tirofiban. These agents have reduced cardiovascular complications in patients undergoing percutaneous coronary intervention (PCI). ${ }^{1-3}$ The differences among these agents lie in the way they bind to the glycoprotein IIb-IIIa receptor. Eptifibatide and tirofiban are ligandmimetic agents, thus inhibiting the binding of fibrinogen to the glycoprotein IIb-IIIa receptor, a critical step in platelet aggregation. Abciximab is a murine-human chimeric monoclonal antibody 
that binds to an epitope on the glycoprotein IIbIIIa complex close to a critical binding site, thus using steric hindrance to inhibit fibrinogen binding.

Glycoprotein IIb-IIIa inhibitors are not devoid of adverse effects, most of which are hematologicminor and major bleeding, anemia, and thrombocytopenia. Severe thrombocytopenia (Table 1) occurs in $0.2-1.2 \%$ of patients treated with eptifibatide or tirofiban and in $1-2 \%$ of those treated with abciximab. ${ }^{1-5}$ In the Platelet Glycoprotein IIb-IIIa in Unstable Angina: Receptor Suppression Using Integrilin Therapy (PURSUIT) trial, eptifibatide was compared with placebo in patients with acute coronary syndrome already receiving heparin and aspirin. ${ }^{4}$ The trial results indicated that morbidity was greater in those who developed thrombocytopenia.

\section{Case Report}

A 62-year-old man presented to the hospital with progression of shortness of breath and fatigue over the last 2 years. He had a history of coronary artery disease and coronary artery bypass graft (left internal mammary artery to the left anterior descending artery, right internal mammary artery to the right coronary artery, and saphenous venous graft to the first obtuse marginal), chronic systolic heart failure, and significant peripheral vascular disease. When the patient was hospitalized approximately 1 year earlier for elective cardiac catheterization, he had significant lesions in the right internal mammary artery graft $(75 \%)$, saphenous venous graft (99\%), and native left main coronary artery (99\%).

It was decided that he would benefit from PCI. However, this procedure was delayed because his serum creatinine concentration was $2.9 \mathrm{mg} / \mathrm{dl}$ (normal range $0.8-1.4 \mathrm{mg} / \mathrm{dl}$ for men), and because of concern that additional contrast dye would increase the risk of worsening renal function.

The patient returned 2 weeks later for hydration before PCI with baseline laboratory values as follows: potassium $4.5 \mathrm{mEq} / \mathrm{L}$ (normal range $3.5-5.0 \mathrm{mEq} / \mathrm{L}$ ), blood urea nitrogen 30 $\mathrm{mg} / \mathrm{dl}(7-21 \mathrm{mg} / \mathrm{dl})$, serum creatinine $2.1 \mathrm{mg} / \mathrm{dl}$, hemoglobin $12.4 \mathrm{~g} / \mathrm{dl}(13.5-17.5 \mathrm{~g} / \mathrm{dl}$ for men), hematocrit $35.9 \%$ (41-53\% for men), and platelet count $127 \times 10^{3} / \mathrm{mm}^{3}\left(150-440 \times 10^{3} / \mathrm{mm}^{3}\right)$. All other laboratory findings were within normal limits. The patient's home drug therapy consisted of aspirin $325 \mathrm{mg} /$ day, carvedilol 12.5
Table 1. Definitions of Thrombocytopenia

\begin{tabular}{ll}
\hline Type of Thrombocytopenia & \multicolumn{1}{c}{ Platelet Count } \\
\hline General definition & $<150 \times 10^{3} / \mathrm{mm}^{3}$ or \\
& $<40 \%$ of baseline count \\
Mild & $100-149 \times 10^{3} / \mathrm{mm}^{3}$ \\
Moderate & $50-99 \times 10^{3} / \mathrm{mm}^{3}$ \\
Severe & $20-49 \times 10^{3} / \mathrm{mm}^{3}$ \\
Profound & $<20 \times 10^{3} / \mathrm{mm}^{3}$ \\
\hline & \multicolumn{1}{c}{ Onset } \\
\hline Acute & $<24$ hrs \\
Chronic & Over days to weeks \\
\hline
\end{tabular}

mg twice/day, losartan $100 \mathrm{mg}$ twice/day, furosemide $80 \mathrm{mg}$ twice/day, potassium chloride $10 \mathrm{mEq} /$ day, digoxin $0.125 \mathrm{mg}$ every other day, felodipine $10 \mathrm{mg}$ twice/day, ezetimide $10 \mathrm{mg} /$ day, atorvastatin $20 \mathrm{mg} /$ day, allopurinol $100 \mathrm{mg} /$ day, colchicine $0.6 \mathrm{mg} / \mathrm{day}$, and sucralfate $1 \mathrm{~g} 4$ times/day, all of which had been part of the patient's long-term treatment regimen.

During catheterization, a sirolimus drugeluting stent was placed in the patient's saphenous venous graft to the first obtuse marginal. The other lesions could not be crossed with a guide wire, so no additional intervention was performed. The patient received intravenous heparin $6000 \mathrm{U}$, a bolus of intravenous abciximab $0.25 \mathrm{mg} / \mathrm{kg}$ followed by 0.125 $\mu \mathrm{g} / \mathrm{kg} / \mathrm{minute}$, and oral clopidogrel $300 \mathrm{mg}$ during the procedure. Clopidogrel $75 \mathrm{mg}$ once/day and abciximab were continued after catheterization.

The patient was transferred to the coronary care unit, where he developed hematemesis 4 hours after the start of heparin and abciximab. Laboratory tests indicated that his hemoglobin level was $11.8 \mathrm{~g} / \mathrm{dl}$, hematocrit $32.5 \%$, and platelet count $7 \times 10^{3} / \mathrm{mm}^{3}$. The aspirin, clopidogrel, and abciximab were discontinued; 2 units of platelets were transfused, and an upper gastrointestinal endoscopy was performed. The endoscopy demonstrated an ulcerative lesion in the stomach, which was treated with an epinephrine injection and an endoscopic ligation clip. Heparin-induced thrombocytopenia was ruled out by the clinical presentation. The patient responded well to the platelet transfusions (Figure 1).

Further review of the patient's medical history revealed that he had experienced a similar episode 11 years earlier. At that time he was admitted with shortness of breath associated with nausea, vomiting, and diaphoresis. Myocardial infarction was ruled out by cardiac enzyme levels 
and electrocardiogram. Three days later he developed chest pain and was enrolled in a clinical trial comparing tirofiban with heparin in patients with unstable angina. The patient's chest pain was relieved after the start of the study drug; however, he developed thrombocytopenia as his platelet count decreased from 372 to $20 \mathrm{x}$ $10^{3} / \mathrm{mm}^{3}$ within 24 hours. The study drug was discontinued and 6 units of platelets were transfused. It was discovered after the study unblinding that the patient had received tirofiban, which was suspected as the cause of the thrombocytopenia (Figure 2).

\section{Discussion}

Thrombocytopenia associated with glycoprotein IIb-IIIa inhibitors has been proposed as being attributable to either pseudothrombocytopenia due to in vitro platelet clumping in blood samples or an immune-mediated reaction. The most notable pathologic mechanism of thrombocytopenia is drug-induced immune thrombocytopenia. Drug-induced immune thrombocytopenia that occurs with glycoprotein IIb-IIIa inhibitors is thought to be due to one of two mechanisms: antibodies recognizing the drug-glycoprotein IIb-IIIa complex or the binding of the drug to the glycoprotein IIb-IIIa receptor, causing a conformational change to the platelet that is recognized by antibodies. ${ }^{6}$ Both ligand-mimetic agents and abciximab have been described with drug-induced immune thrombocytopenia. $^{7}$

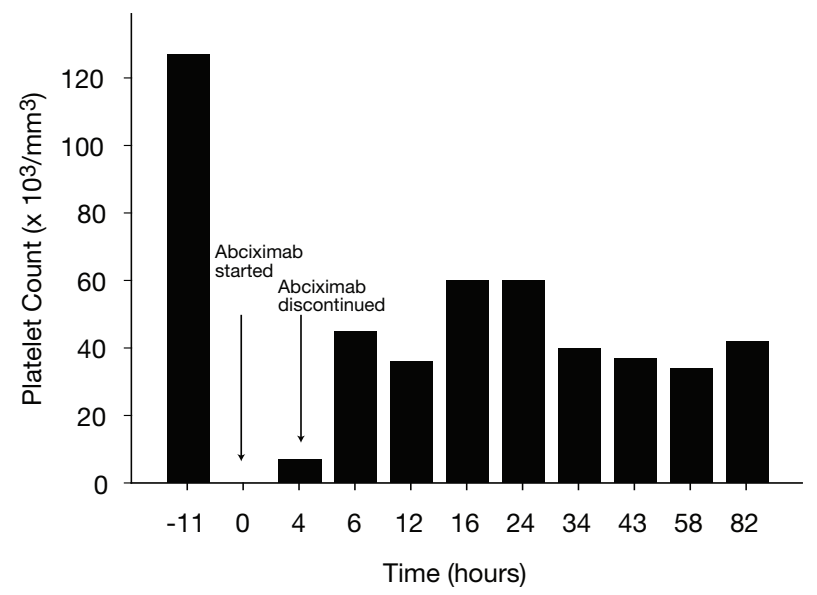

Figure 1. Platelet count recovery in the patient after abciximab was discontinued. Platelet transfusions were given at 5 and 15 hours.
Ligand-Mimetic Agents

A case series of nine patients treated with ligand-mimetic agents (four received tirofiban, five received eptifibatide) demonstrated that the thrombocytopenia was the result of antibodymediated platelet destruction. ${ }^{8}$ The serum of each patient contained a high titer of immunoglobulin $G$ antibody that reacted with the glycoprotein IIb-IIIa complex in the presence of the study drug. Antibodies cross-reacted with other glycoprotein IIb-IIIa inhibitors in only one of the nine patients; no antibodies cross-reacted with abciximab.

\section{Abciximab}

Due to its murine-human chimeric structure, this monoclonal antibody fragment has the potential for antigenicity. Thrombocytopenia with reexposure to abciximab was noted in another case series involving nine patients. ${ }^{9}$ The thrombocytopenia that occurred had a nadir of 6 $\mathrm{x} 10^{3} / \mathrm{mm}^{3}$ or below and took an average of 5 days to return to $100 \times 10^{3} / \mathrm{mm}^{3}$. All nine patients had an antibody that reacted in vitro with platelets coated with abciximab. Those antibodies were distinguished from antibodies reactive to abciximab-coated platelets in healthy individuals. In another case series, four patients experienced acute profound thrombocytopenia (Table 1) within 11-21 hours of receiving abciximab. ${ }^{10}$ Their platelet count remained depressed for 3 days but returned to baseline levels within 2 weeks. The conclusion from both of these case series is that abciximab-induced thrombocytopenia should be considered possible

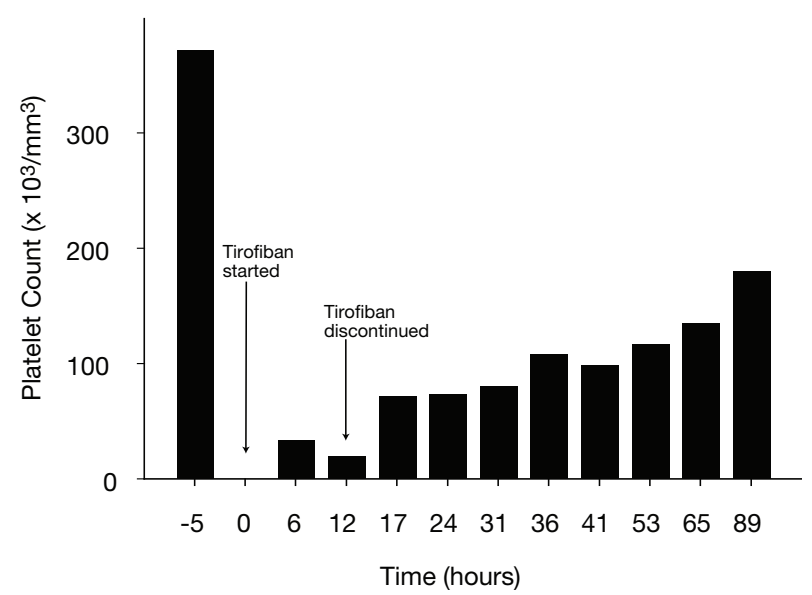

Figure 2. Platelet count recovery in the patient after tirofiban was discontinued. 
in every patient treated despite the time course (at initial exposure or reexposure).

\section{Cross-Reactivity}

Two case reports have shown that patients who develop abciximab-associated thrombocytopenia can safely receive tirofiban or eptifibatide at a later date. ${ }^{11,12}$ Conversely, to our knowledge, there is no documentation of patients who developed thrombocytopenia secondary to tirofiban or eptifibatide having a similar reaction to abciximab. Nor are there data to show crossreactivity from ligand-mimetic agents to abciximab. In fact, abciximab in vitro has inhibited the antibody reaction that causes thrombocytopenia associated with the ligandmimetic agents. ${ }^{8}$ The proposed mechanism for this lack of cross-reactivity is the steric hindrance the molecule provides after binding to the glycoprotein IIb-IIIa receptor.

\section{Management of Drug-Induced Immune} Thrombocytopenia from Glycoprotein IIb-IIIa Inhibitors

The management of drug-induced immune thrombocytopenia from glycoprotein IIb-IIIa inhibitors is not well established. Because further exposure to the drug would perpetuate the reaction, the most common and practical approach is immediate discontinuation of the glycoprotein IIb-IIIa inhibitor. Supportive methods to decrease bleeding and bruising should include avoidance of intramuscular injections and bed rest to decrease the risk of falls. Reported medical management methods are platelet transfusion, and administration of corticosteroids and intravenous immunoglobulin (IGIV). ${ }^{7,8,13-16}$

Platelet transfusion is the most common treatment for thrombocytopenia associated with glycoprotein IIb-IIIa inhibitors. ${ }^{7-9,}$ 13, 15 However, this treatment is controversial. If active drug is still circulating in the body, infused platelets may be destroyed through the same mechanism that destroys endogenous platelets. Platelet infusion may encourage drug binding. This could theoretically decrease the time to platelet recovery. ${ }^{13}$ The National Institutes of Health Consensus Development Conference on Platelet Transfusion Therapy recommends platelet transfusion when the platelet count is below $20 \mathrm{x}$ $10^{3} / \mathrm{mm}^{3}$ or the patient is actively bleeding and has a platelet count below $50 \times 10^{3} / \mathrm{mm}^{3} .^{14}$

Corticosteroid administration in the manage- ment of drug-induced thrombocytopenia has demonstrated mixed results. In a large retrospective review from the Danish Committee on Adverse Drug Reactions, 53\% of 309 patients with noncytotoxic drug-induced thrombocytopenia received treatment with corticosteroids. ${ }^{17}$ No difference was found in platelet count recovery in patients treated with corticosteroids versus those not treated with these agents. However, the review assessed a wide variety of drugs known to induce thrombocytopenia, including those that cause bone marrow suppression. In addition, no cases of glycoprotein IIb-IIIa inhibitor-associated thrombocytopenia were included. Therefore, the review provides little information regarding the value of corticosteroids in treating glycoprotein IIb-IIIa inhibitor-induced thrombocytopenia.

A case report of abciximab-associated thrombocytopenia described combined therapy with platelet transfusions and corticosteroids. ${ }^{15}$ Eight hours after abciximab was started, the patient's platelet count dropped to $10 \times 10^{3} / \mathrm{mm}^{3}$. Abciximab was believed to be the cause of the thrombocytopenia and was discontinued. Intravenous hydrocortisone was started along with the transfusion of 6 units of platelets; 26 hours after abciximab discontinuation, the patient's platelet count rose to greater than $50 \mathrm{x}$ $10^{3} / \mathrm{mm}^{3}$ and hydrocortisone was discontinued. The patient's platelet count dropped precipitously with the discontinuation of hydrocortisone and recovered again when it was restarted. This case report suggests that corticosteroids may be beneficial in the management of thrombocytopenia induced by glycoprotein IIb-IIIa inhibitors. Further investigation should be conducted to confirm this relationship in a larger population of patients with glycoprotein IIb-IIIa inhibitorassociated thrombocytopenia.

For patients with idiopathic thrombocytopenic purpura, IGIV is a therapeutic option, but its role in glycoprotein IIb-IIIa inhibitor-associated immune thrombocytopenia is not well established. The proposed mechanism is that IGIV promotes blockade of the Fc receptors on the macrophage, preventing phagocytosis of the circulating antibody-platelet-glycoprotein IIb-IIIa inhibitor complex. ${ }^{15}$ In a four-patient case series of abciximab-associated acute profound thrombocytopenia, IGIV was given to augment platelet recovery. ${ }^{16}$ Platelet transfusion alone was associated with a rapid platelet recovery $(<4$ hrs). The platelet count recovery did not appear to be augmented by IGIV in two of the four 
patients, and IGIV without platelet transfusion was associated with a very gradual platelet recovery ( $\geq 48 \mathrm{hrs}$ ). This case series involved few patients and thus was limited; however, it demonstrated the efficacy of platelet transfusions and the possible lack of efficacy of IGIV in abciximab-associated immune thrombocytopenia.

\section{Conclusion}

Thrombocytopenia induced by glycoprotein IIb-IIIa inhibitors is a serious and potentially lifethreatening adverse reaction. Although not well understood, the mechanism has been described in many case reports as immune mediated, with both ligand-mimetic agents and abciximab, and with both the start of treatment and reexposure. Our patient's case was unusual in that he developed thrombocytopenia induced by a ligand-mimetic agent 11 years before experiencing a similar reaction to abciximab. The limitation of our conclusion is that our patient was not tested for antibodies, so the thrombocytopenia may have occurred by chance alone. To our knowledge, this case report is the first documentation of a patient with thrombocytopenia associated with both tirofiban and abciximab. This case suggests that such a relationship should be considered when deciding whether to administer abciximab in a patient who has previously developed thrombocytopenia as a reaction to administration of a ligandmimetic glycoprotein IIb-IIIa inhibitor.

\section{References}

1. ESPIRIT Study Investigators. Novel dosing regimen of eptifibatide in planned coronary stent implantation (ESPRIT): a randomized, placebo-controlled trial. Lancet 2000;356: 2037-44.

2. RESTORE Investigators. Effects of platelet glycoprotein IIb/IIIa blockade with tirofiban on adverse cardiac events in patients with unstable angina or acute myocardial infarction undergoing coronary angioplasty. Circulation 1997;96:1445-53.

3. EPIC Investigators. Use of monoclonal antibody directed against the platelet glycoprotein IIb/IIIa receptor in high-risk coronary angioplasty. N Engl J Med 1994;330:956-61.

4. PURSUIT Investigators. Inhibition of platelet glycoprotein IIb/IIIa with eptifibatide in patients with acute coronary syndromes without persistent ST-segment elevation. N Engl J Med 1998;339:436-43.

5. ReoPro Readministration Registry Investigators. Final results of the ReoPro readministration registry. Am J Cardiol 2004;93:979-84.

6. Van den Bemt PL, Meyboom RH, Egberts AC. Drug-induced immune thrombocytopenia. Drug Saf 2004;27:1243-52.

7. Aster RH. Immune thrombocytopenia caused by glycoprotein IIb/IIIa inhibitors. Chest 2005;127:53S-9.

8. Bougie DW, Wilker PR, Wuitschick ED, et al. Acute thrombocytopenia after treatment with tirofiban or eptifibatide is associated with antibodies specific for ligand-occupied GPIIb/IIIa. Blood 2002;100:2071-6.

9. Curtis BR, Swyers J, Divgi A, et al. Thrombocytopenia after second exposure to abciximab is caused by antibodies that recognize abciximab-coated platelets. Blood 2002;99:2054-9.

10. Berkowitz SD, Harrington RA, Rund MM, Tcheng JE. Acute profound thrombocytopenia after c7E3 Fab (abciximab) therapy. Circulation 1997;95:809-13.

11. Desai M, Lucore CL. Uneventful use of tirofiban as an adjunct to coronary stenting in a patient with a history of abciximabassociated thrombocytopenia 10 months earlier. J Invasive Cardiol 2000;12:109-12.

12. Rao J, Mascarenhas DAN. Successful use of eptifibatide as an adjunct to coronary stenting in a patient with abciximabassociated acute profound thrombocytopenia. J Invasive Cardiol 2001;13:471-3.

13. Wazny LD, Ariano RE. Evaluation and management of druginduced thrombocytopenia in the acutely ill patient. Pharmacotherapy 2000;20:292-307.

14. National Institutes of Health. Platelet transfusion therapy. NIH consensus statement online, October 6-8, 1986. Available from www.consensus.nih.gov. Accessed May 23, 2005.

15. Nguyen N, Salib H, Mascarenhas DAN, et al. Acute profound thrombocytopenia without bleeding complications after readministration. J Invasive Cardiol 2001;13:56-8.

16. Kereiakes DJ, Essell JH, Abbottsmith CW, et al. Abciximabassociated profound thrombocytopenia: therapy with immunoglobulin and platelet transfusions. Am J Cardiol 1996;78:1161-3.

17. Pedersen-Njergaard U, Andersen M, Hansen PB. Druginduced thrombocytopenia: clinical data on 309 cases and the effect of corticosteroid therapy. Eur J Clin Pharmacol 1997;52:183-9. 